\title{
Simulation of Heavily Irradiated Silicon Pixel Sensors and Comparison with Test Beam Measurements
}

\author{
Vincenzo Chiochia, IEEE, Member, Morris Swartz, Daniela Bortoletto, Lucien Cremaldi, IEEE, Member, Susanna \\ Cucciarelli, Andrei Dorokhov, Christoph Hörmann, Dongwook Kim, Marcin Konecki, Danek Kotlinski, \\ Kirill Prokofiev, Christian Regenfus, Tilman Rohe, IEEE, Member, David A. Sanders, IEEE, Member, \\ Seunghee Son, Thomas Speer
}

\begin{abstract}
Charge collection measurements performed on heavily irradiated p-spray DOFZ pixel sensors with a grazing angle hadron beam provide a sensitive determination of the electric field within the detectors. The data are compared with a complete charge transport simulation of the sensor which includes signal trapping and charge induction effects. A linearly varying electric field based upon the standard picture of a constant type-inverted effective doping density is inconsistent with the data. A two-trap double junction model implemented in the ISE TCAD software can be tuned to produce a doubly-peaked electric field which describes the data reasonably well. The modeled field differs somewhat $\checkmark$ from previous determinations based upon the transient current technique. The model can also account for the level of charge trapping observed in the data.
\end{abstract}

Index Terms-Pixels; Radiation effects; Space charge; Simulation; Electric fields;

\section{INTRODUCTION}

$\mathbf{I}$ $\mathrm{N}$ the recent years, detectors consisting of one and two dimensional arrays of silicon diodes have come into widespread use as tracking detectors in particle and nuclear physics experiments. It is well understood that the intra-diode electric fields in these detectors vary linearly in depth reaching a maximum value at the $\mathrm{p}-\mathrm{n}$ junction. The linear behavior is a consequence of a constant space charge density, $N_{\text {eff }}$, caused by thermodynamically ionized impurities in the bulk material. It is well known that the detector characteristics are affected by radiation exposure, but it is generally assumed that the same picture is valid after irradiation. In fact, it is

V. Chiochia, C. Regenfus and T. Speer are with the Physik Institut der Universität Zürich, 8057 Zürich, Switzerland.

M. Swartz and D. Kim are with the Johns Hopkins University, Baltimore, MD 21218, USA.

D. Bortoletto and S. Son are with the Purdue University, Task G, West Lafayette, IN 47907, USA.

L. Cremaldi and D.A. Sanders are with the University of Mississippi, Department of Physics and Astronomy, University, MS 38677, USA

S. Cucciarelli and M. Konecki are with the Institut für Physik der Universität Basel, 4056 Basel, Switzerland.

C. Hörmann, A. Dorokhov and K. Prokofiev are with the Physik Institut der Universität Zürich, 8057 Zürich, Switzerland and the Paul Scherrer Institut, 5232 Villigen, Switzerland.

D. Kotlinski and T. Rohe are with the Paul Scherrer Institut, 5232 Villigen, Switzerland. common to characterize the effects of irradiation in terms of a varying effective charge density. The use of $N_{\text {eff }}$ to characterize radiation damage has persisted despite a growing body of evidence [1]- [6] that the electric field does not vary linearly as a function of depth after heavy irradiation but instead exhibits maxima at both $\mathrm{n}^{+}$and $\mathrm{p}^{+}$implants. The work presented in this paper demonstrates conclusively that the standard picture does not provide a good description of irradiated silicon pixel detectors. We show that it is possible to adequately describe the charge collection characteristics of a heavily irradiated silicon detector in terms of a tuned double junction model which produces a doubly-peaked electric field across the detector. The allowed parameter space of the model can also accommodate the expected level of leakage current and the level of charge trapping observed in the detector.

This paper is organized as follows: Section II describes the experimental technique and data, Section III describes the carrier transport simulation used to interpret the data, Section IV describes the technique used to model doubly-peaked electric fields and the limitations of previous models. The tuning of a successful model is discussed in Section V. Section VI summarizes the results and develops several conclusions.

\section{EXPERIMENTAL TECHNique AND DATA}

This investigation is based upon beam test data that were accumulated as part of a program to develop a silicon pixel tracking detector [7] for the CMS experiment at the CERN Large Hadron Collider. The measurements were performed in the H2 line of the CERN SPS in 2003/04 using 150-225 $\mathrm{GeV}$ pions. The beam test apparatus is described in [9] and is shown in Fig. 1. A silicon beam telescope [8] consisted of four modules each containing two $300 \mu \mathrm{m}$ thick single-sided silicon detectors with a strip pitch of $25 \mu \mathrm{m}$ and readout pitch of $50 \mu \mathrm{m}$. The two detectors in each module were oriented to measure horizontal and vertical impact coordinates. A pixel hybrid detector was mounted between the second and third telescope modules on a cooled rotating stage. A trigger signal was generated by a silicon PIN diode. The analog signals from all detectors were digitized in a VME-based readout system 
by two CAEN (V550) and one custom built FADCs. The entire assembly was located in an open-geometry 3T Helmholtz magnet that produced a magnetic field parallel or orthogonal to the beam. The temperature of the test sensors was controlled with a Peltier cooler that was capable of operating down to $-30^{\circ} \mathrm{C}$. The telescope information was used to reconstruct the trajectories of individual beam particles and to achieve a precise determination of the particle hit position in the pixel detector. The resulting intrinsic resolution of the beam telescope was about $1 \mu \mathrm{m}$.

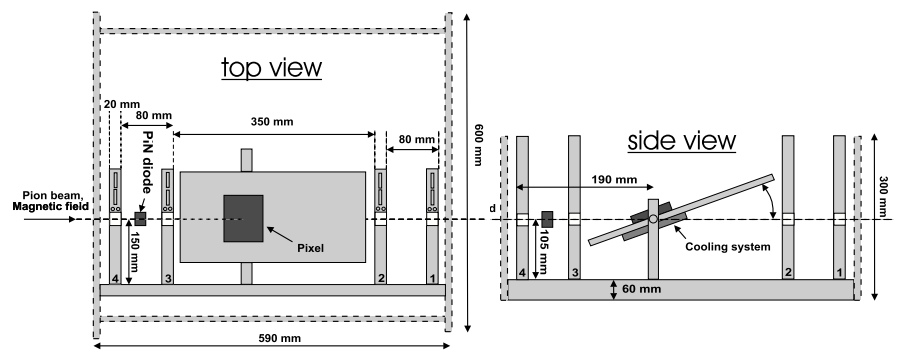

Fig. 1. The beam test apparatus consisting of four horizontal and four vertical planes of silicon strip detectors and a rotating stage for the pixel detector.

\section{A. Pixel Hybrids}

The prototype pixel sensors are so-called "n-in-n" devices: they are designed to collect charge from $\mathrm{n}^{+}$structures implanted into $\mathrm{n}$ - bulk silicon. This design is thought to provide greater longevity in high radiation fields and to allow "partiallydepleted" operation after "type-inversion" of the substrate. It is more expensive than the "p-in-n" process commonly used in strip detectors because it requires double-sided processing and the implementation of inter-pixel isolation. Two isolation techniques were tested: $\mathrm{p}$-spray, where a uniform medium dose of p-impurities covers the whole structured surface, and pstop, where higher dose rings individually surround the $\mathrm{n}^{+}$ implants. Results on the Lorentz angle and charge collection efficiency measurements as well as a detailed description of both designs can be found elsewhere [9], [10]. In this paper we discuss only measurements performed on p-spray sensors. All test devices were $22 \times 32$ arrays of $125 \times 125 \mu \mathrm{m}^{2}$ pixels having a sensitive area of $2.75 \times 4 \mathrm{~mm}^{2}$. The substrate was $285 \mu \mathrm{m}$ thick, n-doped, diffusively-oxygenated silicon of orientation $\langle 111\rangle$ and resistivity $2-5 \mathrm{k} \Omega \cdot \mathrm{cm}$. Individual sensors were diced from fully processed wafers after the deposition of under-bump metalization and indium bumps. A number of sensors were irradiated at the CERN PS with $24 \mathrm{GeV}$ protons. The irradiation was performed without cooling or bias. The applied fluences ${ }^{1}$ were $6 \times 10^{14} \mathrm{n}_{\mathrm{eq}} / \mathrm{cm}^{2}$ and $8 \times 10^{14} \mathrm{n}_{\mathrm{eq}} / \mathrm{cm}^{2}$. The former sample was annealed for three days at $30^{\circ} \mathrm{C}$. In order to avoid reverse annealing, the sensors were stored at $-20^{\circ} \mathrm{C}$ after irradiation and kept at room temperature only for transport and bump bonding. All sensors were bump bonded to PSI30/AC30 readout chips

\footnotetext{
${ }^{1}$ All particle fluences are normalized to $1 \mathrm{MeV}$ neutrons $\left(\mathrm{n}_{\mathrm{eq}} / \mathrm{cm}^{2}\right)$.
}

[11] which allow analog readout of all 704 pixel cells without zero suppression. The PSI30 also has a linear response to input signals ranging from from zero to more than 30,000 electrons.

\section{B. Data}

The main focus of the work presented in this paper involves a set of charge collection measurements that were performed using the "grazing angle technique" [12]. As is shown in Fig. 2, the surface of the test sensor is oriented by a small angle $\left(15^{\circ}\right)$ with respect to the hadron beam. A large sample of data is collected with zero magnetic field and at a temperature of $-10^{\circ} \mathrm{C}$. The charge measured by each pixel along the $y$ direction samples a different depth $z$ in the sensor. Precise entry point information from the beam telescope is used to produce finely binned charge collection profiles. For unirradiated sensors, the cluster length determines the depth over which charge is collected in the sensor.

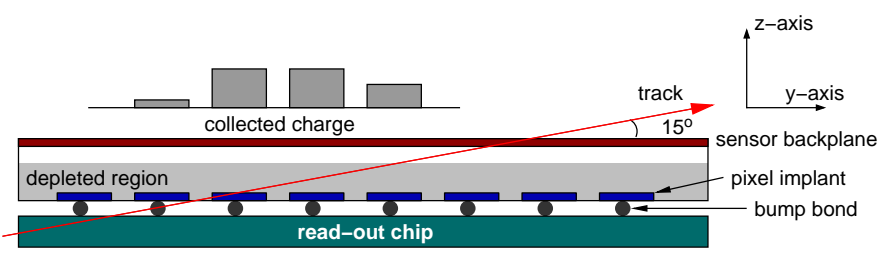

Fig. 2. The grazing angle technique for determining charge collection profiles. The cluster length is proportional to the depth over which charge is collected.

The profiles that were observed for an unirradiated sensor and for a sensor that was irradiated to a fluence of $\Phi=8 \times$ $10^{14} \mathrm{n}_{\mathrm{eq}} / \mathrm{cm}^{2}$ are shown in Fig. 3. The unirradiated sensor was operated at a bias voltage of $150 \mathrm{~V}$ which is well above its depletion voltage (approximately 70V). It produces a large and uniform collected charge distribution indicating that it is fully depleted (a large $y$ coordinate indicates a large collection distance). The irradiated sensor was operated at bias voltages varying from $150 \mathrm{~V}$ to $600 \mathrm{~V}$. It appears to be partly depleted at $150 \mathrm{~V}$, however, signal is collected across the entire thickness of the detector. Another puzzle is that the ratio of charges collected at $300 \mathrm{~V}$ bias and $150 \mathrm{~V}$ bias is 2.1 which is much larger than the maximum value of $\sqrt{2}$ expected for a partly depleted junction. It is clear that the profiles for the irradiated sensor exhibit rather different behavior than one would expect for a heavily-doped, unirradiated sensor.

\section{Simulation AND COMPARISON With DATA}

It is well-known that signal trapping is a significant effect in heavily irradiated silicon detectors. In order to evaluate the effects of trapping, it is necessary to implement a detailed simulation of the sensor. Our simulation, PIXELAV [13], incorporates the following elements: an accurate model of charge deposition by primary hadronic tracks (in particular to model delta rays); a realistic electric field map resulting from the simultaneous solution of Poisson's Equation, carrier continuity equations, and various charge transport models; an 


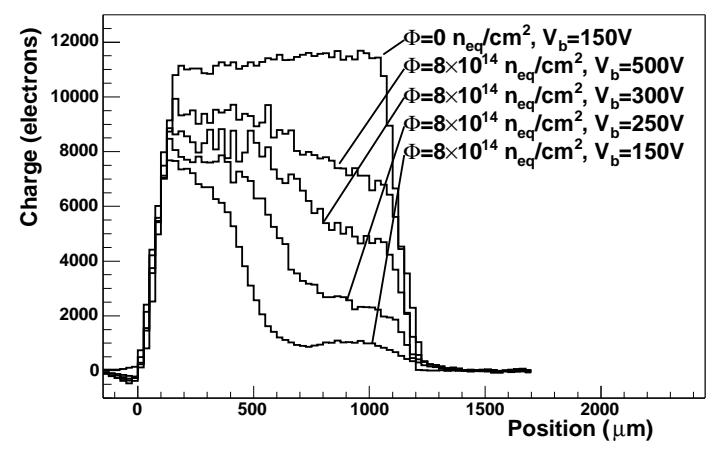

Fig. 3. Charge collection profiles for an irradiated $\left(\Phi=8 \times 10^{14} \mathrm{n}_{\mathrm{eq}} / \mathrm{cm}^{2}\right)$ and an unirradiated sensor $\left(\Phi=0 \mathrm{n}_{\mathrm{eq}} / \mathrm{cm}^{2}\right)$ operated at several bias voltages.

established model of charge drift physics including mobilities, Hall Effect, and 3-D diffusion; a simulation of charge trapping and the signal induced from trapped charge; and a simulation of electronic noise, response, and threshold effects. A final step reformats the simulated data into test beam format so that it can be processed by the test beam analysis software.

Several of the PIXELAV details described in [13] have changed since they were published. The commercial semiconductor simulation code now used to generate a full three dimensional electric field map is the ISE TCAD package [14]. The charge transport simulation was modified to integrate only the fully-saturated drift velocity,

$$
\frac{d \vec{r}}{d t}=\frac{\mu\left[q \vec{E}+\mu r_{H} \vec{E} \times \vec{B}+q \mu^{2} r_{H}^{2}(\vec{E} \cdot \vec{B}) \vec{B}\right]}{1+\mu^{2} r_{H}^{2}|\vec{B}|^{2}}
$$

where $\mu(\vec{E})$ is the mobility, $q= \pm 1$ is the sign of the charge carrier, $\vec{E}$ is the electric field, $\vec{B}$ is the magnetic field, and $r_{H}$ is the Hall factor of the carrier. The use of the fullysaturated drift velocity permits much larger integration steps (which had previously been limited by stability considerations) and significantly increases the speed of the code.

The simulation was checked by comparing simulated data with measured data from an unirradiated sensor. A plot of the charge measured in a single pixel as a function of the horizontal and vertical track impact position for normally incident tracks is shown in Fig. 4. The simulation is shown as the solid histogram and the test beam data are shown as solid points. Note that the sensor simulation does not include the "punch-through" structure on the $\mathrm{n}^{+}$implants which is used to provide a high resistance connection to ground and to provide the possibility of on-wafer IV measurements. There is reduced charge collection from this portion of the implant and the data shows reduced signal in both projections at the bias dot. Another check, shown in Table I, is the comparison of the average Lorentz angle measured at several bias voltages [9]. In both cases, reasonable agreement is observed between measured and simulated data.

The charge collection profiles for a sensor irradiated to a fluence of $\Phi=6 \times 10^{14} \mathrm{n}_{\text {eq }} / \mathrm{cm}^{2}$ and operated at bias voltages
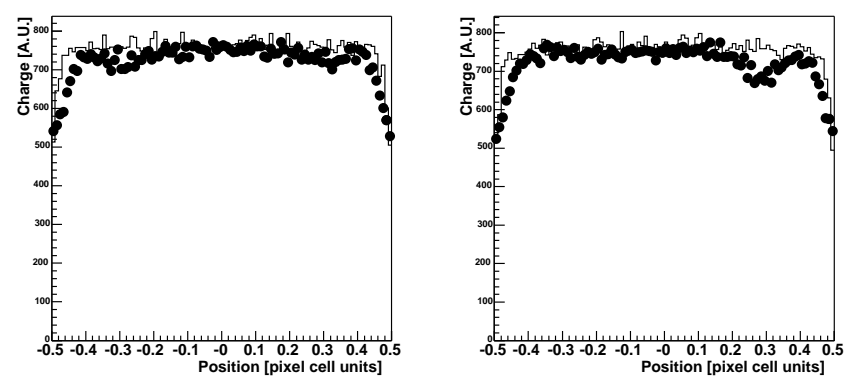

Fig. 4. Collected charge measured in a single pixel as a function of the horizontal (left) and vertical (right) track impact position for tracks that are normally incident on an unirradiated sensor. The simulation is shown as a solid histogram and the test beam data are shown as solid dots.

TABLE I

Measured AND Simulated VALUES of AVERAGE LORENTZ ANGLE $\theta_{L}$ VERSUS BIAS VOLTAGE FOR AN UNIRRADIATED SENSOR.

\begin{tabular}{|ccc|}
\hline Bias Voltage & Measured $\theta_{L}[\mathrm{deg}]$ & Simulated $\theta_{L}[\mathrm{deg}]$ \\
\hline $150 \mathrm{~V}$ & $22.8 \pm 0.7$ & $24.7 \pm 0.9$ \\
$300 \mathrm{~V}$ & $14.7 \pm 0.5$ & $17.4 \pm 0.9$ \\
$450 \mathrm{~V}$ & $11.2 \pm 0.5$ & $12.0 \pm 0.9$ \\
\hline
\end{tabular}

of $150 \mathrm{~V}$ and $300 \mathrm{~V}$ are presented in Fig 5. The measured profiles are shown as solid dots and the simulated profiles are shown as histograms. The simulated profiles were generated with electric field maps corresponding to two different effective densities of acceptor impurities. The full histograms are the simulated profile for $N_{\text {eff }}=4.5 \times 10^{12} \mathrm{~cm}^{-3}$. Note that the $300 \mathrm{~V}$ simulation reasonably agrees with the measured profile but the $150 \mathrm{~V}$ simulation is far too broad. The dashed histograms show the result of increasing $N_{\text {eff }}$ to $24 \times 10^{12} \mathrm{~cm}^{-3}$. At this effective doping density, the width of the simulated peak in the $150 \mathrm{~V}$ distribution is close to correct but it does not reproduce the "tail" observed in the data at large $y$. The $300 \mathrm{~V}$ simulated distribution is far too narrow and the predicted charge is lower than the data (note that the profiles are absolutely normalized). It is clear that a simulation based upon the standard picture of a constant density of ionized acceptor impurities cannot reproduce the measured profiles.

Note that the simulation of this irradiated sensor includes the effects of trapping. The trapping rates of electron and holes have been shown to scale linearly with fluence [15], [16]. Unfortunately, the measured fluences have a fractional uncertainty of $\pm 10 \%$ which feeds directly into the uncertainty on the trapping rates. Additional uncertainty arises because annealing can modify the trapping rates by $30 \%$ [15] leading to a fairly large overall uncertainty.

\section{TWO-TRAP MODELS}

The large number of measurements suggesting that large electric fields exist at both sides of an irradiated silicon diode has given rise to several attempts to model the effect [3], [6], [17]. The most recent of these by Eremin, Verbitskaya, and Li 

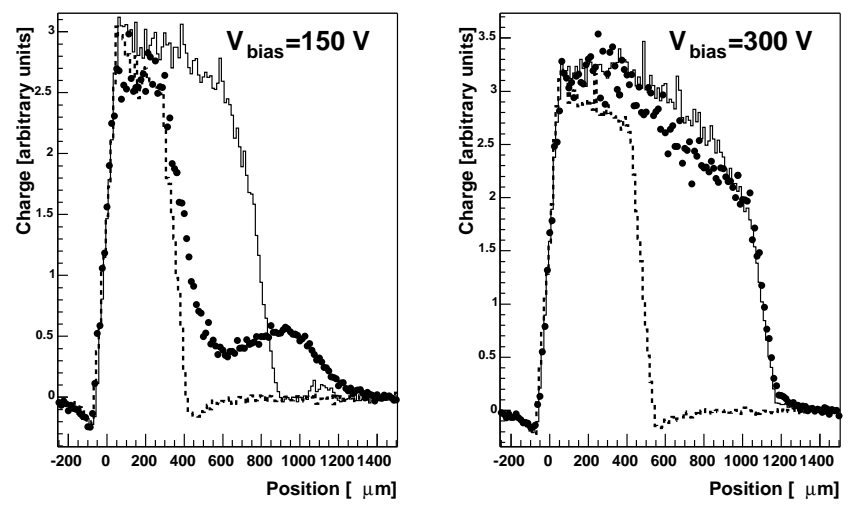

Fig. 5. The measured and simulated charge collection profiles for a sensor irradiated to a fluence of $\Phi=6 \times 10^{14} \mathrm{n}_{\mathrm{eq}} / \mathrm{cm}^{2}$. The profiles measured at bias voltages of $150 \mathrm{~V}$ and $300 \mathrm{~V}$ are shown as solid dots. The full histograms are the simulated profiles for a constant effective doping $N_{\text {eff }}=4.5 \times 10^{12} \mathrm{~cm}^{-3}$ of acceptor impurities. The dashed histograms are the simulated profiles for a constant effective doping $N_{\text {eff }}=24 \times 10^{12} \mathrm{~cm}^{-3}$.

(EVL) [17] is based upon a modification of the Shockley-ReadHall (SRH) statistics. The EVL model produces an effective space charge density $\rho_{\text {eff }}$ from the trapping of leakage current by one acceptor trap and one donor trap. The effective charge density is related to the occupancies and densities of traps as follows,

$$
\rho_{\text {eff }}=e\left[N_{D} f_{D}-N_{A} f_{A}\right]+\rho_{\text {dopants }}
$$

where: $N_{D}$ and $N_{A}$ are the densities of donor and acceptor trapping states, respectively; $f_{D}$ and $f_{A}$ are the occupied fractions of the donor and acceptor states, respectively, and $\rho_{\text {dopants }}$ is the charge density due to ionized dopants. Charge flows to and from the trapping states due to generation and recombination. The occupied fractions are given by the following standard SRH expressions,

$$
\begin{aligned}
f_{D} & =\frac{v_{h} \sigma_{h}^{D} p+v_{e} \sigma_{e}^{D} n_{i} e^{E_{D} / k T}}{v_{e} \sigma_{e}^{D}\left(n+n_{i} e^{E_{D} / k T}\right)+v_{h} \sigma_{h}^{D}\left(p+n_{i} e^{-E_{D} / k T}\right)}(3) \\
f_{A} & =\frac{v_{e} \sigma_{e}^{A} n+v_{h} \sigma_{h}^{A} n_{i} e^{-E_{A} / k T}}{v_{e} \sigma_{e}^{A}\left(n+n_{i} e^{E_{A} / k T}\right)+v_{h} \sigma_{h}^{A}\left(p+n_{i} e^{-E_{A} / k T}\right)}
\end{aligned}
$$

where: $v_{e}$ and $v_{h}$ are the thermal speeds of electrons and holes, respectively; $\sigma_{e}^{D}$ and $\sigma_{h}^{D}$ are the electron and hole capture cross sections for the donor trap; $\sigma_{e}^{A}$ and $\sigma_{h}^{A}$ are the electron and hole capture cross sections for the acceptor trap; $n$ and $p$ are the densities of free electrons and holes, respectively; $n_{i}$ is the intrinsic density of carriers; $E_{D}$ and $E_{A}$ are the activation energies (relative to the mid-gap energy) of the donor and acceptor states, respectively. The generation-recombination current caused by the SRH statistics for single donor and acceptor states is given by the following expression,

$$
\begin{aligned}
U & =\frac{v_{h} v_{e} \sigma_{h}^{D} \sigma_{e}^{D} N_{D}\left(n p-n_{i}^{2}\right)}{v_{e} \sigma_{e}^{D}\left(n+n_{i} e^{E_{D} / k T}\right)+v_{h} \sigma_{h}^{D}\left(p+n_{i} e^{-E_{D} / k T}\right)} \\
& +\frac{v_{h} v_{e} \sigma_{h}^{A} \sigma_{e}^{A} N_{A}\left(n p-n_{i}^{2}\right)}{v_{e} \sigma_{e}^{A}\left(n+n_{i} e^{E_{A} / k T}\right)+v_{h} \sigma_{h}^{A}\left(p+n_{i} e^{-E_{A} / k T}\right)} .(4)
\end{aligned}
$$

Within the EVL model, the four trapping cross sections are set to $10^{-15} \mathrm{~cm}^{2}$. The leakage current is generated from an additional SRH trapping state that is introduced for this purpose but is assumed not to trap charge. The donor and acceptor states are assumed not to generate leakage current which, given the small size of the cross sections, is a self-consistent assumption. The densities of the donor and acceptor states $\left(N_{D}\right.$ and $\left.N_{A}\right)$ are adjusted to "fit" TCT data. The parameters of the model are given in Table II. The trap densities are scaled to fluence and are given in terms of introduction rates $g_{\mathrm{int}}=N_{A / D} / \Phi_{\text {eq }}$. An illustrative sketch of the EVL model has been reproduced

TABLE II

PARAMETERS OF THE EVL MODEL [17].

\begin{tabular}{|ccccc|}
\hline Trap & $\mathrm{E}(\mathrm{eV})$ & $g_{\text {int }}\left(\mathrm{cm}^{-1}\right)$ & $\sigma_{e}\left(\mathrm{~cm}^{2}\right)$ & $\sigma_{h}\left(\mathrm{~cm}^{2}\right)$ \\
\hline Donor & $E_{V}+0.48$ & 6 & $1 \times 10^{-15}$ & $1 \times 10^{-15}$ \\
Acceptor & $E_{C}-0.525$ & 3.7 & $1 \times 10^{-15}$ & $1 \times 10^{-15}$ \\
\hline
\end{tabular}

from [17] and is shown in Fig. 6. Figure 6a shows a uniform current density flowing across a reverse-biased junction. Since holes are produced uniformly across the junction and flow to the $\mathrm{p}^{+}$backplane, the hole current density increases linearly with increasing $z$ from the $\mathrm{n}^{+}$implant to the $\mathrm{p}^{+}$implant. The electrons flow to the $\mathrm{n}^{+}$implant and the electron current density increases with decreasing $z$. The actual carrier densities depend upon the details of the fields and mobilities but vary monotonically across the junctions as shown in Fig. 6b. The trapping of the mobile carriers produces a net positive space charge density near the $\mathrm{p}^{+}$backplane and a net negative space charge density near the $\mathrm{n}^{+}$implant as shown in Fig. 6c. Since positive space charge corresponds to n-type doping and negative space charge corresponds to p-type doping, there are p-n junctions at both sides of the detector. The electric field in the sensor follows from a simultaneous solution of Poisson's equation and the carrier continuity equations. The resulting $z$ component of the electric field is shown in Fig. 6d. It varies with an approximately quadratic dependence upon $z$ having a minimum at the zero of the space charge density and maxima at both implants.

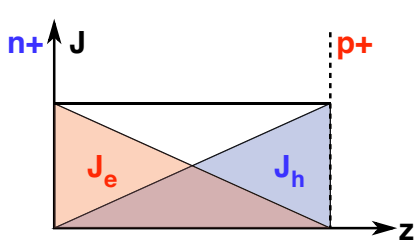

(a)

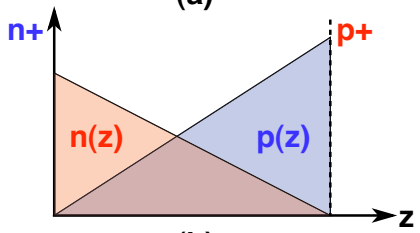

(b)

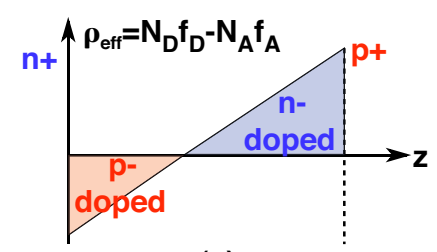

(c)

(d)

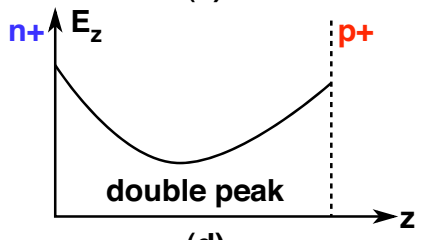

Fig. 6. An illustrative sketch of the EVL model [17]. 
In order to test whether the electric field predicted by the EVL model would improve the agreement between the simulated and measured charge collection profiles shown in Section III, it was necessary to implement the EVL model in ISE TCAD. TCAD contains a complete implementation of SRH statistics. However, the EVL modifications of SRH are not incorporated. In particular, any state added to generate leakage current would also trap charge. It is possible to use the TCAD "Physical Model" interface to replace the existing SRH implementation with a modified one, however, a less invasive approach was adopted.

Our approach is based upon three observations:

1) The trapping cross sections are poorly known. The cross sections for states observed in various types of defect spectroscopy vary over several orders of magnitude.

2) The occupancies $f_{D / A}$ of the trapping states are independent of the scale of the cross sections. If the capture cross sections $\sigma_{e / h}$ in Eq. 3 are rescaled by a factor $r$, then $f_{D / A}$ are unchanged. The occupancies depend only upon the ratio of electron and hole cross sections, $\sigma_{h} / \sigma_{e}$.

3) The current $U$ generated by the donor and acceptor impurities is linear in the cross section rescaling factor $r$.

These observations imply that it is possible to implement the EVL model in TCAD simply by setting $\sigma_{e}^{D}=\sigma_{h}^{D}=\sigma_{e}^{A}=\sigma_{h}^{A}$ and by varying the size of the common cross section until the generation current is equal to the observed or expected leakage current. The trap occupancies are not affected in zeroth order by the rescaling, but the leakage current and the free carrier densities are affected by $r$. The carrier densities have a firstorder effect on the occupancies so that varying $r$ does alter $\rho_{\text {eff }}$. This approach uses the same trapping states to produce space charge and leakage current (it is not necessary to introduce current-generating states).

Using the activation energies and introduction rates for the donor and acceptor states given in Table II, the only free parameter in the TCAD implementation of the EVL model is the size of the common cross section or equivalently, the leakage current. The leakage current in the test sensors was observed to increase substantially after bump-bonding to the readout chips. Although the cause of the increased current is not clear, it is possible that it is due to increased surface/edge leakage or that it is caused by stressing the detector. The measured leakage current, expressed in terms of the damage parameter $\alpha$ [18], is listed in Table III. It is well established that in large detectors, the magnitude of leakage current is expected to be $\alpha_{0} \simeq 4 \times 10^{-17} \mathrm{~A} / \mathrm{cm}$ for a fully biased detector [18]. The observed leakage current is approximately five times larger than the expected value.

The EVL model was implemented in the sensor simulation using several parameter choices. The resulting charge collection profiles for a sensor irradiated to a fluence of $\Phi=8 \times$ $10^{14} \mathrm{n}_{\mathrm{eq}} / \mathrm{cm}^{2}$ and operated at $150 \mathrm{~V}$ and $300 \mathrm{~V}$ bias voltages are shown in Fig. 7. The measured profiles are again shown as solid dots. The dotted histogram shows the EVL model with
TABLE III

OBSERVED LEAKAGE CURRENT IN TERMS OF $\alpha=I_{\text {leak }}\left(20^{\circ} \mathrm{C}\right) /(\mathcal{V} \Phi)$ WHERE: $I_{\text {leak }}\left(20^{\circ} C\right)$ IS THE LEAKAGE CURRENT EXPECTED AT TEMPERATURE $20^{\circ} \mathrm{C}, \mathcal{V}$ IS THE VOLUME OF THE SENSOR, AND $\Phi$ IS THE NEUTRON-EQUIVALENT FLUENCE.

\begin{tabular}{|cc|}
\hline Bias Voltage & $\alpha(\mathrm{A} / \mathrm{cm})$ \\
\hline$-150 \mathrm{~V}$ & $15 \times 10^{-17}$ \\
$-300 \mathrm{~V}$ & $19 \times 10^{-17}$ \\
$-450 \mathrm{~V}$ & $25 \times 10^{-17}$ \\
\hline
\end{tabular}

the leakage current adjusted to $20 \%$ of the measured value which is comparable to the expected leakage current $\left(\alpha_{0}\right)$. It clearly substantially underestimates the collected charge at both voltages. The effect of increasing the leakage current to $60 \%$ of the measured value is shown as the dashed histogram. This improves the agreement at $300 \mathrm{~V}$ but produces too much signal in the high $z$ tail of the $150 \mathrm{~V}$ distribution. Finally, in an attempt to increase the collected charge at $300 \mathrm{~V}$, the introduction rates were scaled down by a factor of eight. The common cross section was increased by the same factor to hold the leakage current constant. The result of this is shown as the solid histogram in Fig. 7. Note that this does increase the collected charge signal, however, it is still below the data at $300 \mathrm{~V}$ and is much too large in the tail region of the $150 \mathrm{~V}$ distribution. We conclude that the EVL model does not provide a quantitative description of the measured charge collection profiles.
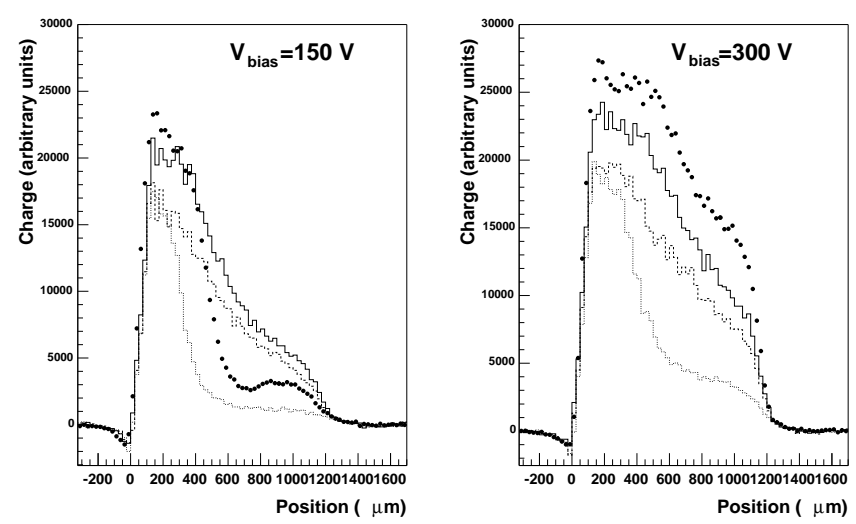

Fig. 7. The measured charge collection profiles for a sensor irradiated to a fluence of $\Phi=8 \times 10^{14} \mathrm{n}_{\mathrm{eq}} / \mathrm{cm}^{2}$ (solid dots) and operated at $150 \mathrm{~V}$ and $300 \mathrm{~V}$ are compared with simulations based upon the EVL model. The dotted histogram shows the EVL model with the leakage current adjusted to $20 \%$ of the measured value which is comparable to the expected leakage current $\left(\alpha_{0}\right)$. The dashed histogram shows the effect of increasing the leakage current to $60 \%$ of the measured value. The solid histogram shows the result of decreasing the introduction rates by a factor of eight and increasing the cross sections by the same factor.

\section{AN IMPRoved Two-Trap Model}

Although it led to a poor quantitative description of the measured charge collection profiles, the EVL model does have the qualitative features needed to describe the data. At low 
bias voltages, the combination of the quadratic minimum in the electric field and signal trapping can act like a "gate" suppressing the collection of charge from the $\mathrm{p}^{+}$side of the detector. The measured profile would then appear to be a narrow peak on the $\mathrm{n}^{+}$side of the detector. As the bias is increased, the magnitude of the field at the minimum would also increase and effectively "lift the gate" which would allow much more charge collection from the $\mathrm{p}^{+}$side of the detector.

In order to investigate whether a two-trap double junction EVL-like model can describe the measured charge collection profiles, a tuning procedure was adopted. Relaxing the EVL requirement that all trapping cross sections are equal, the model has six free parameters $\left(N_{D}, N_{A}, \sigma_{e}^{D}, \sigma_{h}^{D}, \sigma_{e}^{A}, \sigma_{h}^{A}\right)$ that can be adjusted. The activation energies are kept fixed to the EVL values. Additionally, as was discussed in Section III, the electron and hole signal trapping rates, $\Gamma_{e}$ and $\Gamma_{h}$, are uncertain at the $30 \%$ level due to the fluence uncertainty and possible annealing of the sensors. They are treated as constrained parameters. The parameters of the double junction model were systematically varied and the agreement between measured and simulated charge collection profiles was judged subjectively.

In the course of the tuning procedure, it became clear that the EVL model does not produce a sufficiently large electric field on the $\mathrm{p}^{+}$side of the detector. The solution to this problem is to increase the density of donors (hole traps) as compared to the density of acceptors (electron traps). When this is done, the $z$ position of the minimum in the effective charge density shifts toward the $\mathrm{n}^{+}$implant as sketched in Fig. 8a. Unfortunately, this causes the "peak" in the 150V simulated charge profile to become too narrow. The position of the charge density minimum can be restored to a position nearer the midplane of the detector by decreasing the ratios of the hole and electron cross sections from 1.0 to $0.25\left(\sigma_{h}^{D} / \sigma_{e}^{D}=0.25\right.$ and $\left.\sigma_{h}^{A} / \sigma_{e}^{A}=0.25\right)$ as shown schematically in Fig. 8b. Note that the adjustment of the cross sections for both trap types minimizes the field in the quadratic minimum while allowing for large fields at the implants. For simplicity it is assumed that the electron cross sections are equal $\left(\sigma_{e}^{D}=\sigma_{e}^{A}=\sigma_{e}\right)$ and that the hole cross sections are equal $\left(\sigma_{h}^{D}=\sigma_{h}^{A}=\sigma_{h}\right)$.
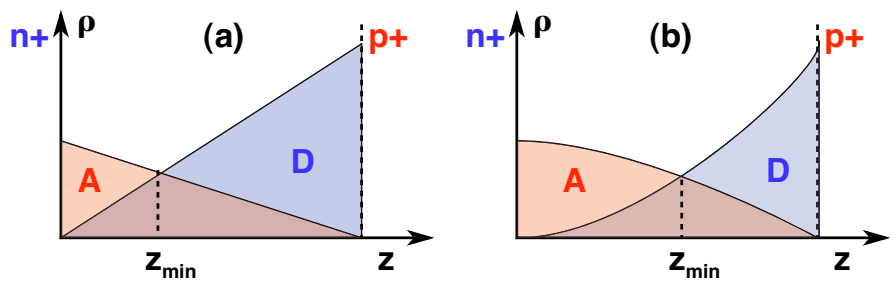

Fig. 8. The effect of increasing $N_{D} / N_{A}$ when (a) the electron and hole cross sections are equal, and when (b) $\sigma_{h} / \sigma_{e}=0.25$.

The current best "fit" to the measured charge collection profiles is called model dj44 and reduces the ratio between the densities of acceptor and donor states, $N_{A} / N_{D}$, from the EVL value of 0.62 to 0.40 . The $z$-component of the simulated electric field, $E_{z}$, is plotted as a function of $z$ in Fig. 9 for bias voltages of $150 \mathrm{~V}$ and $300 \mathrm{~V}$. The field profiles have minima near the midplane of the detector. Note that the minimum field at $150 \mathrm{~V}$ bias appears to be very small but is still approximately $400 \mathrm{~V} / \mathrm{cm}$. The electric field profiles resulting from a constant p-type doping of density $N_{\text {eff }}=4.5 \times 10^{12} \mathrm{~cm}^{-3}$ are shown as dot-dashed and dotted curves for reference.

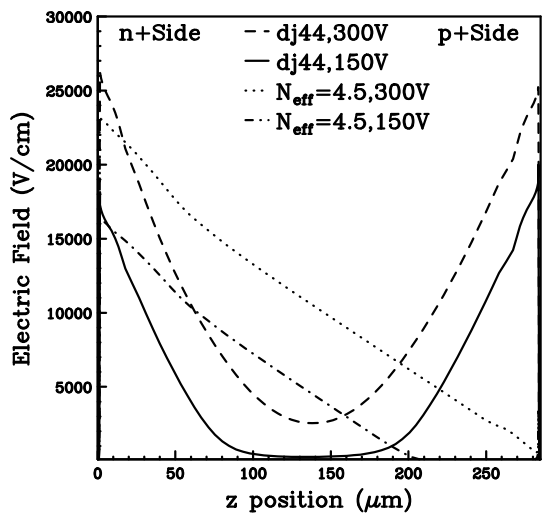

Fig. 9. The $z$-component of the simulated electric field resulting from model dj44 is plotted as a function of $z$. The field profiles for $150 \mathrm{~V}$ and $300 \mathrm{~V}$ are shown as as solid and dashed curves, respectively. The electric field profiles resulting from a constant p-type doping of density $N_{\text {eff }}=4.5 \times 10^{12} \mathrm{~cm}^{-3}$ are shown as dot-dashed and dotted curves for $150 \mathrm{~V}$ and $300 \mathrm{~V}$, respectively.

The measured charge collection profiles at bias voltages between $150 \mathrm{~V}$ and $450 \mathrm{~V}$ are compared with the dj44 simulation in Fig. 10 for a fluence of $6 \times 10^{14} \mathrm{n}_{\mathrm{eq}} / \mathrm{cm}^{2}$. The electron trapping rate for the lower fluence is set to $93 \%$ of the nominal value.

Although for high values of the bias voltage the simulation falls below the measured profile, it provides a reasonable description of the measurements. Several features of the measured distributions are described well by the simulation. Note that both data and simulation show a distinctly negative signal near $y=0 \mu \mathrm{m}$. This can be understood as a consequence of hole trapping. Electrons deposited near the $\mathrm{n}^{+}$implant are collected with high efficiency whereas holes deposited near the implant must transit the entire detector thickness to reach the $\mathrm{p}^{+}$ backplane. If the holes are collected, they produce no net signal on the $\mathrm{n}^{+}$side of the detector. However if the holes trap, then a negative signal is induced and is most visible in the $y<0 \mu \mathrm{m}$ region. Another feature is the "wiggle" in the $150 \mathrm{~V}$ profiles. The relative signal minimum near $y=700 \mu \mathrm{m}$ corresponds to the $E_{z}$ minimum where both electrons and holes travel only short distances before trapping. This small separation induces only a small signal on the $\mathrm{n}^{+}$side of the detector. At larger values of $y, E_{z}$ increases causing the electrons drift back into the minimum where they are likely to trap. However, the holes drift into the higher field region near the $\mathrm{p}^{+}$implant and are more likely to be collected. The net induced signal on the $\mathrm{n}^{+}$ side of the detector therefore increases and creates the local maximum seen near $y=900 \mu \mathrm{m}$.

The dj44 model fixes the ratios $N_{A} / N_{D}$ and the ratio 

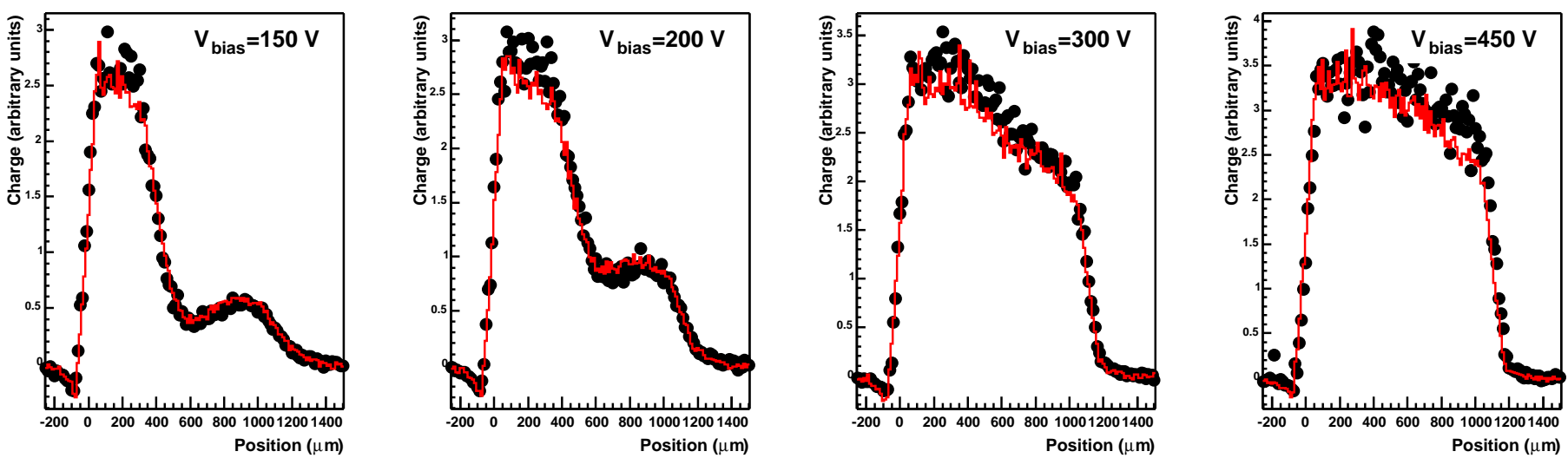

Fig. 10. The measured charge collection profiles at bias voltages of $150 \mathrm{~V}, 200 \mathrm{~V}, 300 \mathrm{~V}$, and $450 \mathrm{~V}$ are shown as solid dots for fluences of $6 \times 10^{14} \mathrm{n}_{\mathrm{eq}} / \mathrm{cm}^{2}$. The dj44 simulation is shown as the solid histogram in each plot.

between cross sections $\sigma_{h} / \sigma_{e}$ leaving the parameters $N_{D}$ and $\sigma_{e}$ to vary. Over a restricted range, an increase in $N_{D}$ can be offset by a decrease in $\sigma_{e}$ keeping the electric field profile approximately unchanged. Scaling the electron cross section as $\sigma_{e} \propto N_{D}^{-2.5}$ produces very similar charge collection profiles. The allowed region in the $N_{D}-\sigma_{e}$ space is shown in Fig. 11a as the solid line in the logarithmic space. If the donor density becomes too small $\left(N_{D}<20 \times 10^{14} \mathrm{~cm}^{-3}\right)$, the $150 \mathrm{~V}$ simulation produces too much signal at large $z$. If the donor density becomes too large $\left(N_{D}>50 \times 10^{14} \mathrm{~cm}^{-3}\right)$, the $300 \mathrm{~V}$ simulation produces insufficient signal at large $z$. Since the simulated leakage current varies as $I_{\text {leak }} \propto \sigma_{e} N_{D}$, different points on the allowed solid contour correspond to different leakage current. Contours of constant leakage current are shown as dashed curves and are labeled in terms of the corresponding damage parameter $\alpha$ where $\alpha_{0}$ is the expected leakage current. It is clear that the simulation can accommodate the expected leakage current which is smaller than the measured current by a factor of three.

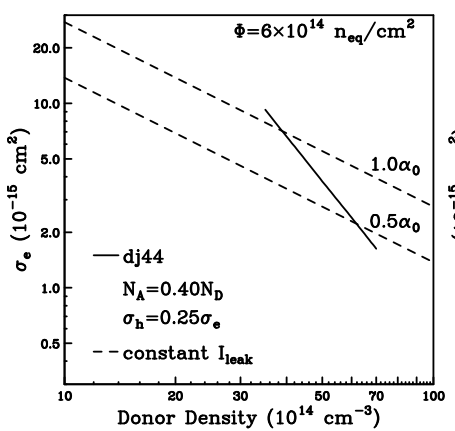

(a)

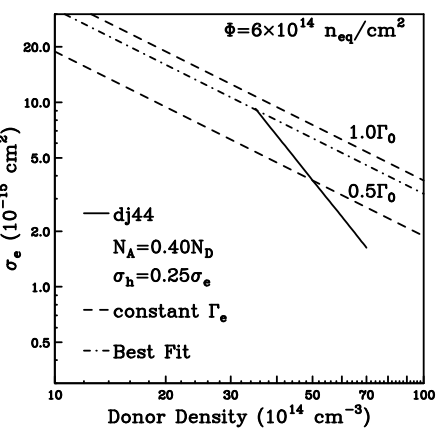

(b)
Fig. 11. The allowed region in the $N_{D}-\sigma_{e}$ space for model dj44 is shown as the solid line in (a) and (b). Contours of constant leakage current are shown as dashed curves in (a) and are labeled in terms of the corresponding damage parameter $\alpha$ where $\alpha_{0}$ is the expected leakage current. Contours of constant electron trapping rate are shown as dashed curves in (b) and are labeled in terms of the un-annealed trapping rate $\Gamma_{0}$ for the nominal fluence.
The electron and hole traps in the model should also contribute to the trapping of signal carriers. The contributions of these states to the effective trapping rates of electrons and holes are given by the following expressions

$$
\begin{aligned}
\Gamma_{e} & =v_{e}\left[\sigma_{e}^{A} N_{A}\left(1-f_{A}\right)+\sigma_{e}^{D} N_{D} f_{D}\right] \simeq v_{e} \sigma_{e}^{A} N_{A} \\
\Gamma_{h} & =v_{h}\left[\sigma_{h}^{D} N_{D}\left(1-f_{D}\right)+\sigma_{h}^{A} N_{A} f_{A}\right] \simeq v_{h} \sigma_{h}^{D} N_{D}
\end{aligned}
$$

where it has been assumed that the trap occupancies are small. Because $N_{A} / N_{D}$ is assumed to be constant, contours of constant electron trapping rate are parallel to contours of constant leakage current in $N_{D^{-}} \sigma_{e}$ space. The best "fit" of the simulation to the measured profiles reduced $\Gamma_{e}$ to $93 \%$ of the un-annealed trapping rate $\Gamma_{0}$ for the nominal fluence [15]. These contours are compared with the allowed contour in Fig. 11b. It is clear that the simulation can accommodate the measured trapping rate in the same region of parameter space that maximizes the leakage current.

Figure $11 \mathrm{~b}$ also suggests a solution to the puzzle that the trapping rates have been shown to be unaffected by the presence of oxygen in the detector bulk [15] whereas it is well-established that the space charge effects are quite sensitive to the presence of oxygen in the material [19]. It is clear from Fig 11b that small-cross-section trapping states can play a large role in the effective charge density but a small one in the effective trapping rates: every point on the dj44 line produces $100 \%$ of the effective charge density but only the larger cross section points contribute substantially to the trapping rate. If the formation of the additional small-cross-section states were suppressed by oxygen, then $\rho_{\text {eff }}$ could be sensitive to oxygenation whereas $\Gamma_{e / h}$ would be insensitive to oxygenation. This is another consequence of the observation that the occupancies $f_{D / A}$ of the trapping states are independent of the scale of the cross sections in the steady state (see Section IV). The trapping of signal carriers is not a steady-state phenomenon and is sensitive to the scale of the trapping cross sections. 


\section{CONCLUSIONS}

The main result of the work presented in this paper is that a doubly-peaked electric field is necessary to describe the charge collection profiles measured in heavily irradiated pixel sensors. A simulation utilizing a linearly varying electric field based upon the standard picture of a constant type-inverted effective charge density is inconsistent with the measurements.

A two-trap EVL-like model can be tuned to provide a reasonable description of the measurements. It can also account for the expected level of the leakage current (although not the observed leakage current) and the observed electron signal trapping rate. It is important to state that any two-trap model is, at best, an "effective theory". It is well-known that there are many trapping states in heavily irradiated silicon that trap charge. There may also be thermodynamically ionized defects that contribute to the effective space charge density. Clearly a two-trap model can describe the gross features of the physical processes in our sensors but it may not be able to describe all details. This also implies that the parameters of the two-trap model presented in this paper are unlikely to have physical reality.

The charge-sharing behavior and resolution functions of many detectors are sensitive to the details of the internal electric field. A known response function is a key element of any reconstruction procedure. A working effective model will permit the detailed response of these detectors to be tracked as they are irradiated in the next generation of accelerators.

Finally, we note that despite a growing body of contrary evidence, the overly simple picture of uniform type inversion in irradiated silicon detectors persists in the minds of many researchers and even in the terminology used by experts to describe their devices. After irradiation, quantities like $\ell_{\text {dep }}$ (depletion depth) and $N_{\text {eff }}$ may correctly suggest reduced detector performance but given the reality of doubly-peaked electric fields and signal trapping, they have no physical significance.

\section{REFERENCES}

[1] Z. Li and H.W. Kramer, "Fast neutron radiation effects in silicon detectors fabricated by different thermal oxidation processes", IEEE Trans. Nucl. Sci., 39, pp. 577-583, 1992.

[2] L.J. Beattie, T.J. Brodbeck, A. Chilingarov, G. Hughes, S.A. McGarry, et al., "The electric field in irradiated silicon detectors", Nucl. Instr. Meth., A418, pp. 314-321, 1998.

[3] G. Casse, M. Glaser, and E. Grigoriev, "Study of evolution of active volume in irradiated silicon detectors", Nucl. Instr. Meth., A426, pp. 140146, 1999.

[4] C. Leroy, P. Roy, G. Casse, M. Glaser, E. Grigoriev and F. Lemeilleur, et al., "Study of charge transport in non-irradiated and irradiated silicon detectors", Nucl. Instr. Meth., A426, pp. 99-108, 1999.

[5] D. Menichelli, M. Bruzzi, Z. Li, and V. Eremin, "Modelling of observed double-junction effect", Nucl. Instr. Meth., A426, p. 135-139, 1999.

[6] A. Castaldini, C. Canali, A. Cavallini, F. Nava, and L. Polenta, "Doublejunction effect in proton-irradiated silicon diodes", Journal of Applied Physics 92, pp. 2013-2016, 2002.

[7] The CMS Collaboration, "CMS Tracker", CERN, Geneva, Switzerland, Technical Design Report LHCC98-6, 1988.

[8] C. Amsler, K. Bsiger, M. Glttli, R. Kaufmann, F. Ould-Saada, et al., "A high resolution silicon beam telescope", Nucl. Inst. Meth., A480, pp. 501-507, 2002.
[9] A. Dorokhov, C. Amsler, D. Bortoletto, V. Chiochia, L. Cremaldi, et al., "Tests of silicon sensors for the CMS pixel detector", Nucl. Inst. Meth. A530, pp. 71-76, 2004.

[10] T. Rohe, D. Bortoletto, V. Chiochia, L.M. Cremaldi, S. Cucciarelli, et al., "Position dependence of charge collection in prototype sensors for the CMS pixel detector", IEEE Trans. Nucl. Sci. 51, pp. 1150-1157, 2004.

[11] D. Meer, "Bau und Messen eines Multichip Pixelmodules als Prototyp für den CMS-Tracker", Diplomarbeit, Eidgenössische Technische Hochschule, Zürich, Switzerland, March 2000.

[12] B. Henrich, W. Bertl, K. Gabathuler, R. Horisberger, "Depth profile of signal charge collected in heavily irradiated silicon pixels", CMS Note 1997/021, March 1997. [Online] Available: http://cmsdoc.cern.ch/doc/notes/docs/NOTE1997_021.

[13] M. Swartz, "CMS pixel simulations", Nucl. Instr. Meth., A511, pp. 88-91, 2003.

M. Swartz, "A Detailed Simulation of the CMS Pixel Sensor", CMS Note 2002/027, July 2002. [Online]. Available: http://cmsdoc.cern.ch/doc/notes/docs/NOTE2002_027.

[14] ISE-TCAD, Integrated System Engineering AG, Zürich, Switzerland.

[15] G. Kramberger, et al., ROSE/TN/2001-01, January 2001.

G. Kramberger, V. Cindro, I. Mandic, M. Mikuz and M. Zavrtanik, et al., "Determination of effective trapping times for electrons and holes in irradiated silicon", Nucl. Inst. Meth., A476, pp. 645-651, 2002.

[16] O. Krasel, et al., presented at the 3rd RD50 Workshop, CERN, Geveva, 2003. [Online]. Available: http://rd50.web.cern.ch/rd50/3rdworkshop/talks/2-4-Olaf-Krasel.pdf.

[17] V. Eremin, E. Verbitskaya, and Z. Li, "The origin of double peak electric field distribution in heavily irradiated silicon detectors", Nucl. Instr. Meth., A476, pp. 556-564, 2002.

[18] M. Moll, E. Fretwurst, and G. Lindström, "Leakage current of hadron irradiated silicon detectors - material dependence", Nucl. Instr. Meth., A426, pp. 87-93, 1999.

[19] G. Lindström, "Radiation damage in silicon detectors", Nucl. Instr. Meth., A512, pp. 30-43, 2003.

S. Dittongo, L. Bosisio, M. Ciacchi, D. Contarato, G. D'Auria, et al., "Radiation hardness of different silicon materials after high-energy electron irradiation", Nucl. Instr. Meth., A530, pp. 110-116, 2004. 